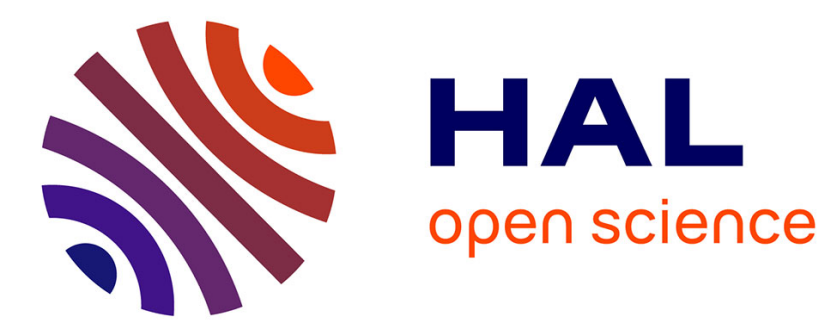

\title{
Populations animales et systèmes agraires. L'exemple des bovins laitiers
}

B. Vissac

\section{To cite this version:}

B. Vissac. Populations animales et systèmes agraires. L'exemple des bovins laitiers. Productions Animales, 1994, 7 (2), pp.97-113. hal-00896079

\section{HAL Id: hal-00896079 https://hal.science/hal-00896079}

Submitted on 1 Jan 1994

HAL is a multi-disciplinary open access archive for the deposit and dissemination of scientific research documents, whether they are published or not. The documents may come from teaching and research institutions in France or abroad, or from public or private research centers.
L'archive ouverte pluridisciplinaire HAL, est destinée au dépôt et à la diffusion de documents scientifiques de niveau recherche, publiés ou non, émanant des établissements d'enseignement et de recherche français ou étrangers, des laboratoires publics ou privés. 
INRA Prod. Anim., 1994, 7 (2), 97 - 113

\section{B. VISSAC}

INRA Département Systèmes agraires et Développement

147 rue de l'Université

75338 Paris Cedex 07

\section{Populations animales et systèmes agraires. L'exemple des bovins laitiers}

ports au territoire sont peu pris en compte sauf sous l'angle de leur acception économique globale (bassins de production). La crise correspond par ailleurs à des atteintes jugées excessives à l'environnement : émission de polluants ici et, son corollaire, abandon du territoire rural là. Aux liens existants au sein de ces deux facettes de la crise : quantité vs qualité pour les filières, pollution vs abandon pour l'environnement, apparaissent des liens entre elles. L'intensification excessive n'est pas indépendante de la pollution et la déprise agricole n'est pas nécessairement synonyme de qualité des produits. Traiter ces atteintes à l'environnement par des mesures spécifiques, définies en termes généraux, s'avère souvent plus coûteux que de modifier le mode de gestion des élevages et des filières de production dans le sens d'une utilisation plus équilibrée des territoires et des rentes culturelles qui leur sont associées.

\section{Résumé}

Cette étude vise à appréhender la crise des rapports entre la Société et son territoire dans le domaine de l'élevage à travers sa double traduction : excédents quantitatifs et déficits qualitatifs des productions d'une part, perceptions sociales d'atteintes à l'environnement d'autre part. L'élevage bovin laitier est considéré comme un bon support de réflexion car il est un témoin privilégié du modernisme en élevage. La notion de système agraire apparaît appropriée à la prise en compte de cette complexité. Celle de population animale, considérée dans sa double identité, biologique et culturelle, est utilisée comme un marqueur du système agraire.

Dans la première partie, on étudie, au cours des deux derniers siècles, l'évolution des populations bovines traites, sur le territoire national, avec celle des systèmes agraires supports. On s'appuie, dans ce cadre, sur une périodisation correspondant aux trois entités utilisées successivement pour définir les populations : populations locales, organisations raciales, souches issues de schémas collectifs de sélection.

On dégage ensuite, dans une deuxième partie, les traits principaux de cette évolution : élargissement territorial et structuration des organisations sociales avec la spécialisation; stratification régionale des populations plus évoluées sur les plus anciennes à un rythme fonction des possibilités de transformation des systèmes agraires ; diffusion des nouveaux types de populations autour de pôles émergeant des marges des anciennes structures spatiales. On constate que cette dynamique à long terme de délocalisation des populations bovines laitières s'accompagne de résistances locales des anciennes populations, résistances qui sont avivées par la crise.

Cette contradiction dans l'évolution actuelle de l'élevage bovin laitier conduit à élargir la réflexion sur un métadéveloppement dans le sens des idées développées par Morin. On en discute, sur quelques exemples, les aspects bioécologiques, psychologiques et socio-culturels et leurs relations. On suggère leur intégration dans un processus de Recherche-Action associant les partenaires des fillières de production à ceux qui ont en charge la gestion du territoire rural et où les populations animales peuvent représenter des indicateurs d'une culture collective.

Ces ouvertures visent à approfondir le champs d'application des approches pluridisciplinaires locales fondées sur les postulats de globalité et de complexité des systèmes agraires à base d'élevage. Elles peuvent fournir des éléments de réflexion pour un renouveau de la loi sur l'élevage de 1964 dans lequel la population animale garderait une place prééminente. 
Prendre en compte cette double complexité en intégrant la gestion des filières de production qui sont au coeur des préoccupations des zootechniciens avec celle des territoires qui les supportent, et auxquels ils s'intéressent moins dans ce sens, suppose de fonder le raisonnement sur un concept intégrateur. Celui de système agraire (Cholley 1946, Vissac et Hentgen 1979, Mazoyer 1985) et les développements récents dont il a été l'objet à travers la modélisation systémique (Brossier et al 1990) semblent convenir pour aborder cette complexité dans une perspective utile au développement agricole et rural (Vissac et Benoit 1993). Ce concept est fondé sur la prise en compte des pratiques mises en oeuvre par les exploitants à titre individuel et collectif à travers les choix des techniques et leur mise en oeuvre au niveau conjoint des filières et des territoires aménagés pris dans la continuité de ces aménagements.

Mais, le système agraire est difficile à identifier directement à travers des indicateurs qui soient pertinents vis à vis des filières et des territoires et qui rejoignent les représentations qu'en ont les acteurs du développement rural. Certains, comme Deffontaines, ont recours aux effets des techniques agricoles sur le territoire en explicitant leurs rapports au paysage. On peut aussi envisager de s'intéresser à la combinaison des techniques mises en oeuvre par les agriculteurs (système de pratiques selon Cristofini et al 1978). Certaines de ces combinaisons techniques mettent en jeu des organisations collectives (systèmes socio-techniques) mais peuvent s'exprimer à travers des indicateurs simples dépassant l'objet que représentent ces indicateurs : c'est le cas de la population animale (Vissac 1978). Cette dernière est considérée à la fois sous son contenu d'information (définition génétique) et comme un objet de pratiques collectives d'élevage et de sélection (définition de nature anthropologique). Ces deux définitions sont liées : la première traduit un effet et la deuxième en constitue la cause.

L'objet de ce texte qui fait suite à une présentation auprès d'un public élargi (Vissac 1993) est d'étudier, sur un cas particulier, les correspondances entre les populations animales et leurs systèmes agraires supports. Legay (1993), commentant ce texte, distingue trois grandes périodes dans l'Histoire des relations de l'Homme avec les autres êtres vivants : la période de domestication largement empirique et inconsciente (révolution néolithique) ; celle, dominée par une sélection consciente associée à de fortes interactions avec le milieu, qui connut son apogée au début du siècle dernier ; celle enfin qui débute à peine où, contrairement aux précédentes, les connaissances scientifiques acquises au niveau cellulaire et moléculaire précédent la pratique. Il remarque justement que si, sous l'impulsion du progrès de ces connaissances, beaucoup de recherches ont été consacrées aux périodes extrêmes, on connaît peu de choses sur la deuxième période qui est pourtant celle dans laquelle nous gérons l'élevage actuellement. Cela s'explique, en grande partie, par l'absence d'informations fiables et par la stagnation apparente des conceptions existantes, accessibles aux éleveurs et adaptées à leur situation.

Nous nous intéresserons donc au cas particulier des populations bovines utilisées pour la production de lait, depuis le début du siècle dernier, époque à partir de laquelle on commence à disposer d'informations et de règles d'action validables en matière de sélection. Le choix de ces populations s'explique par le fait qu'elles constituent des témoins privilégiés de la mise en oeuvre du modernisme en élevage à travers l'intensification et la spécialisation laitière.

Le texte comporte trois parties : l'étude des relations entre populations bovines traites et systèmes agraires et de leur évolution depuis 1800 , l'identification d'invariants ou de lois d'évolution conjointe, l'application de cette réflexion au contexte de la crise actuelle de l'élevage bovin laitier dans ses rapports aux filières et aux territoires.

\section{1 / Dynamique conjointe des populations et des systèmes agraires}

Les généticiens font classiquement référence à trois phases dans l'évolution des populations animales au cours des deux siècles écoulés. Ils se fondent sur leur mode de désignation : la phase des populations locales désignées par des noms de lieux ou de produits laitiers (Gilibert 1991), celle des races à standard spécifié puis celle des lignées intraraciales découlant de l'application de schémas de sélection collectifs dont l'identification correspond à des index génotypiques pour des caractères de production.

\section{1 / Le temps des lieux}

Le début du siècle dernier correspond donc à l'apogée des organisations paysannes locales ; elles développaient une agriculture à base de subsistance fondée sur des relations de proximité. La sélection empirique était intégrée aux pratiques agraires de ces organisations et générait des réseaux d'échange de reproducteurs qui participaient fortement à leur structuration sociale.

Les objectifs de sélection variaient d'une population à une autre suivant la gamme de leurs usages dans les milieux et des pratiques d'élevage qui leur y étaient appliquées. Dans une agriculture basée sur les rapports aux ressources locales, on conçoit, par ailleurs, que de faibles différences de milieux et de risques climatiques puissent se traduire fortement au niveau des populations animales. Les formes de traction animale ont très tôt conduit à distinguer les populations bovines du Nord de celles du Sud de la France : dans le Nord, le contexte agronomique plus favorable a conduit à adopter la traction chevaline et orienté l'élevage des bovins vers les productions de lait et de viande sous des formes d'élevage limitant les effets des aléas. Dans le Sud, au contraire, 
la traction bovine et le poids des influences du milieu ont généré des populations plus diverses dans leurs aptitudes de production et d'adaptation.

La pression variable de la sélection naturelle et de la sélection inconsciente (adaptation aux pratiques des éleveurs), qui favorisait surtout la fertilité et la viabilité, se conjuguait avec des oppositions entre ces caractères d'une part et les caractères de production de lait et de viande d'autre part pour identifier les populations locales. Ces populations correspondaient ainsi chacune à des états d'équilibre particuliers des gènes propres à de petites régions géographiques. Compte tenu de l'état limité des connaissances, les éleveurs mélangeaient ainsi, sous le vocable de "génération", des effets génétiques, embryologiques avec les influences d'un "milieu" qui, par delà leurs pratiques, étaient associées au contexte géographique. Il n'y a donc rien d'étonnant au fait qu'aient émergé, dans cette situation, des croyances renvoyant aux formes de l'imaginaire local.

Les populations locales ainsi identifiées et que l'on peut qualifier de prédarwiniennes ne subsistent que dans des situations insulaires où l'isolement, la spécificité des conditions d'élevage et l'adversité du milieu les ont protégées des effets des phénomènes de migration.

\section{2 / Le temps des races}

La phase raciale trouve ses fondements à travers des opérations de rationalisation de l'élevage pour la production de lait autour de foyers industriels en émergence dans le Lancashire : c'est, selon Russell (1986), derrière des conceptions parfois extravagantes, la première mention d'une plus value des animaux indépendante de leur propre valeur productive. Cette initiative a été prolongée par des éleveurs contemporains et proches de Darwin ; elle n'a connu son plein épanouissement que dans le cadre de l'élevage ovin dont le rythme de reproduction, la taille des troupeaux et le rôle dans le système d'innovations agraires en émergence dans les Midlands constituaient des avantages incontestables (Bakewell, cité par Russell 1986) par rapport aux bovins que sélectionnait Collins, cité par Russell (1986), dans la vallée de la Tees. Une catégorie de commerçants aristocratiques était en train de naître.

Cette dynamique qui concernait surtout les troupeaux allaitants était fondée sur un projet productif commun que favorisait l'apparition de systèmes agraires régionaux basés sur des échanges marchands à plus grand rayon d'action. L'information à la base de la sélection correspondait surtout, en l'absence de mesures objectives qui sont intervenues plus tard (début du $20^{\text {eme }}$ siècle en France), à des critères de coloration du pelage et de morphologie corporelle supposés liés aux aptitudes productives. Les concours de bétail organisés par les Sociétés Départementales d'Agriculture qui étaient impulsées par des nobles, en rupture de cour, et des bourgeois terriens, en quête de reconnaissance sociale dans les campagnes, constituaient, en France, le «fer de lance» de ce mouvement qui participait, plus largement, de l'«anglomanie-agromanie» vers le milieu du $19^{\text {ème }}$ siècle. Le poids excessif accordé dans la sélection à l'ascendance, parfois lointaine, rejoignait la tradition du respect des grandes familles et participait à la permanence de la structure sociale de ces sélectionneurs qui concentraient les ufonctions de juge et de marchand" (Pluvinage).

Les livres généalogiques des principales races françaises sont apparus dans cette mouvance ; les populations locales situées dans les contextes agronomiques les plus favorables, les plus ouverts aux échanges et sous l'effet des sélectionneurs les plus dynamiques, absorbant les populations locales avoisinantes dès lors qu'aucune spécificité naturelle ou culturelle ne s'y opposait. Leur création date de l'époque du «mélinisme» (fin du siècle passé), le rôle de l'administration visait surtout à élargir la base sociale quand elle existait ou à la constituer ailleurs (création des syndicats d'élevage dans l'Est où se manifestait, à travers les "fruitières", une tradition coopérative de transformation fromagère). Les modèles développés par cette base étaient supposés diffusables à la masse des éleveurs.

Les grandes races laitières (Normande, Montbéliarde et Pie Noire) ont ainsi émergé logiquement des zones à traction chevaline sous l'influence d'organisations économiques valorisant la production de lait et son sousproduit boucher à travers des rentes culturelles ou de localisation : la proximité du marché parisien a été longtemps essentielle, avant que ne se développe la congélation des produits. On remarque également que ces races ont pris naissance dans le pourtour du territoire national où le siège de leur «livre généalogique» s'est constamment maintenu pendant cette phase de l'évolution (Caen, Besançon et Cambrai respectivement). Cette remarque conduit à souligner le rôle de l'influence étrangère sur ces phénomènes par le biais des courants d'idées et d'introductions d'animaux de la part de migrants novateurs. La Normandie était sous l'emprise de courants d'échanges avec l'Angleterre ; la Montbéliarde a bénéficié du dynamisme des mennonites suisses dans la maîtrise de l'élevage et de la filière des fromages de garde ; la Frisonne Pie Noire ne s'est implantée que plus récemment, par la Thiérache et les Ardennes, qui constituaient les lieux de passage du cheptel des Pays-Bas considéré comme prévalent en matière de rendement laitier. Le herd-book Français Frison Pie Noir n'a été créé que plus récemment.

La figure 1 illustre l'émergence des trois grandes races laitières et traduit l'état de leur expansion géographique à la fin de la phase du développement racial, vers 1950 : la zone centrale, où dominent les races allaitantes, et la bordure méditerranéenne d'où l'élevage bovin avait disparu à cette époque, sont éliminées de la représentation.

Cette identification raciale sera suffisamment forte pour résister à la phase suivante.

\section{Le système agraire est un concept intégrateur de ces 2 dimensions et la population animale en est un indicateur dans sa double définition, biologique et sociale.}


Figure 1. Dynamique d'exploitation territoriale des populations bovines laitières.

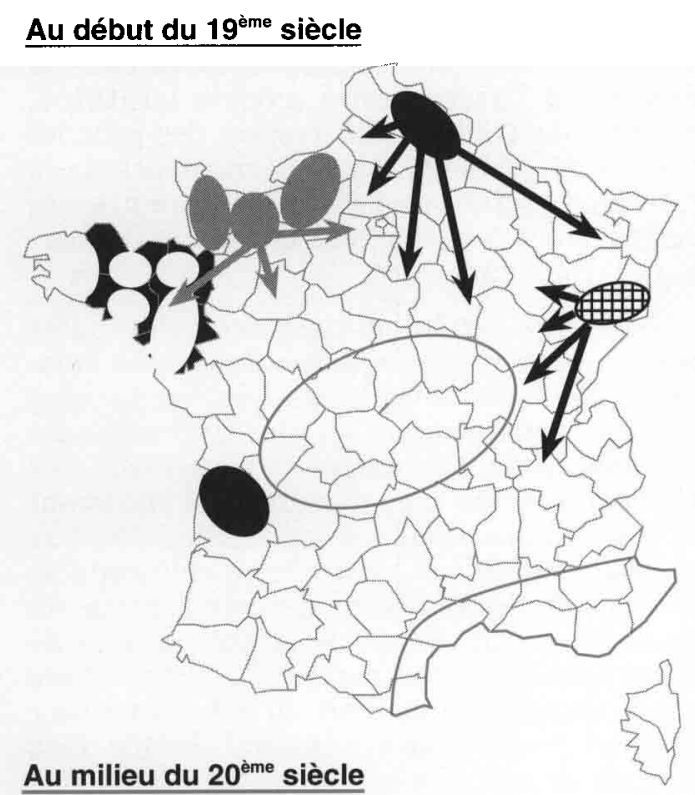

\section{Après les prémices de la sélection rationnelle et la primauté de l'ascendance est arrivé l'âge d'or des génotypes spécialisés.}

\section{3 / Le temps des schémas}

Contrairement à l'Angleterre, la France s'est opposée au processus de spécialisation qui était inadapté à ses systèmes agraires paysans (Geffroy 1978). Au lendemain de la dernière guerre toutefois, la volonté de modernisation de l'agriculture (suppression de la traction animale et spécialisation des exploitations) s'imposait au plan politique. L'émergence de la génétique quantitative et les progrès dans la maitrise de la reproduction offraient alors la possibilité de jeter les bases d'une sélection collective sur les caractères de production. Elle imposait de facto, par une limitation du nombre de caractères, une spécialisation poussée des races. Cette sélection collective était le «fer de lance» d'une organisation qui devait transformer un élevage bovin national peu productif mais gros consommateur d'aides électoralistes qui en faisaient un véritable «tonneau des danaïdes» des fonds publics. L'esprit coopératif et mutualiste dominait alors et il était censé permettre l'introduction des nouvelles techniques dans des exploitations de taille plus modeste par rapport à celles de nos principaux concurrents : l'élevage laitier, qui était adapté à ce type d'exploitations et pour lequel un caractère simple de sélection dominait constituait la cible idéale de ces schémas collectifs de sélection en direction des jeunes agriculteurs. Tel était l'objectif et le cadre d'application de la loi sur l'élevage qui s'intégrait et inspirait une organisation générale des services professionnels du Développement.

Les index génotypiques des reproducteurs pour le critère de sélection, le niveau génétique des populations et de leurs composantes pour ce critère (troupeau, ensemble des troupeaux d'une zone donnée...) constituaient les nouvelles identifications de ces populations qui se sont constituées à l'intérieur des races.

Bien que l'objectif de la production poussait à une maîtrise intensive et à la spécialisation laitière, les schémas de sélection ont respecté au départ, sauf rare exception (Norvège par exemple), le critère de race ; ils ont épousé des configurations territoriales mouvantes au gré des alliances des centres d'insémination. Puis, l'émergence d'un grand marché communautaire et la concurrence internationale ont accéléré la constitution de grands bassins de collecte laitière, dans le Grand Ouest surtout ; ils ont favorisé l'expansion de la Frisonne Pie Noire dont le siège a quitté Cambrai pour la Bretagne et l'infusion de gènes Holstein en son sein (nouvelle dénomination de Prim'Holstein). Cette infusion, plus ou moins contrôlée, est également intervenue dans les Pies Rouges laitières sous le camouflage psychologique nécessaire du gène rouge de coloration du pelage présent en Pie Noire.

Vingt cinq ans après le début d'application des schémas de sélection, on peut mesurer l'efficacité productive des schémas sur le critère de sélection dominant : la quantité de matières utiles du lait par lactation (en kilogrammes). La figure 2 illustre les résultats correspondants par race et par département. 
Figure 2. Potentiel génétique des populations raciales. Le niveau génétique est celui de la cohorte des génisses nées en 1988 . II est exprimé après diverses corrections d'effets non génétiques, en déviation par rapport à un niveau de base 0 correspondant à l'époque où ont commencé à se manifester les effets de la loi sur l'élevage sur l'ensemble du territoire national (1973).

Pie-Noire

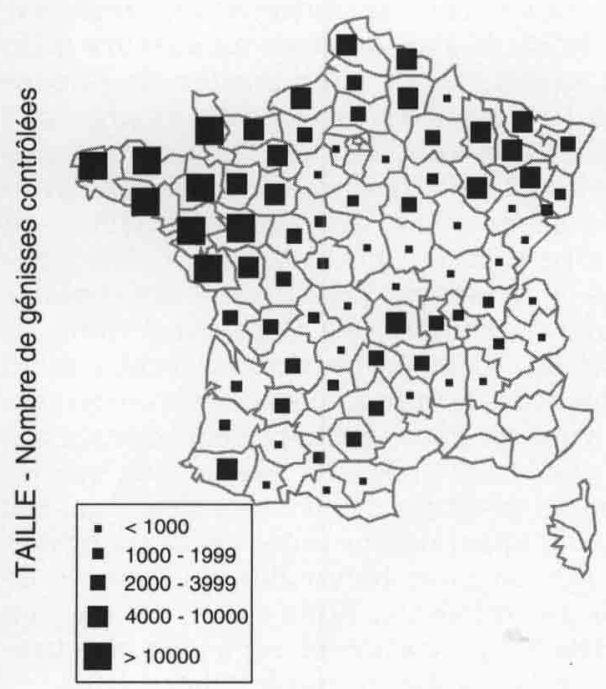

을

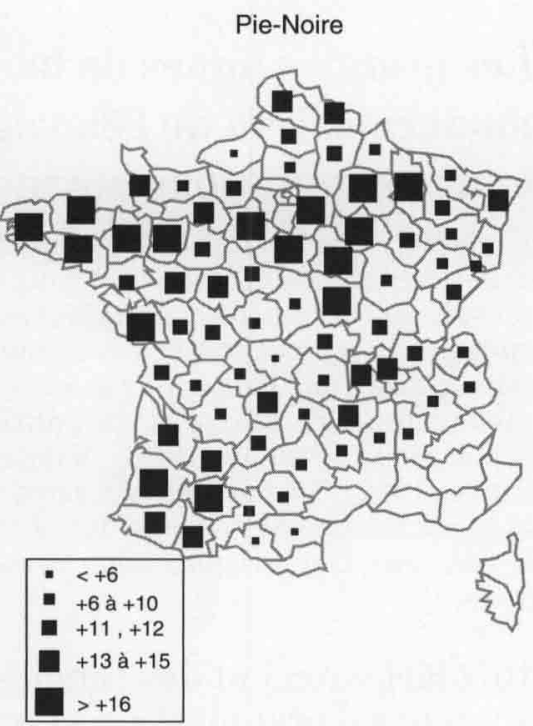

Pie-Noire

을

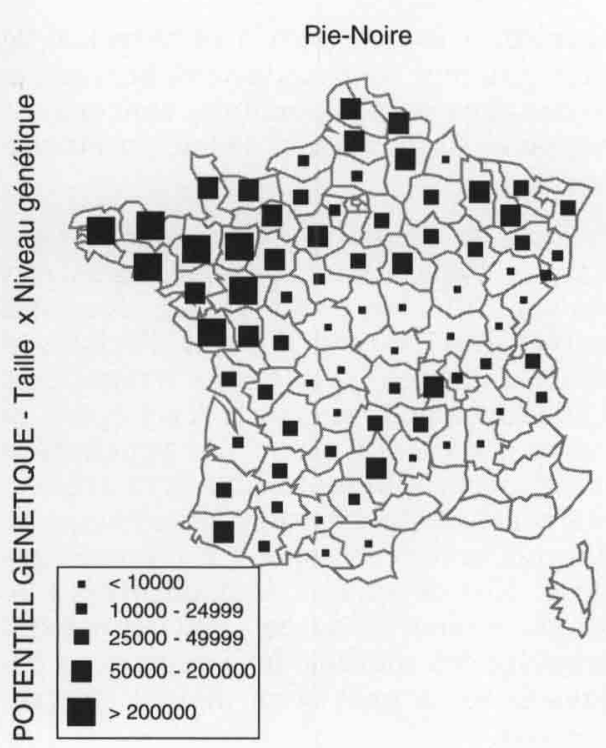

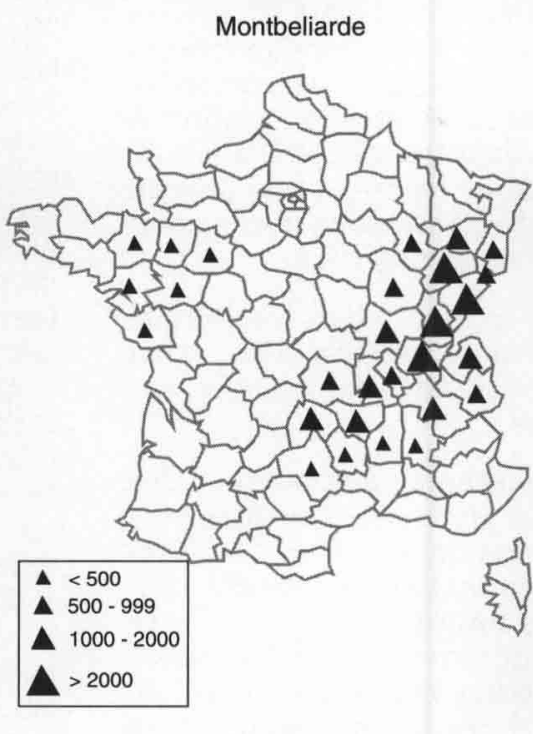

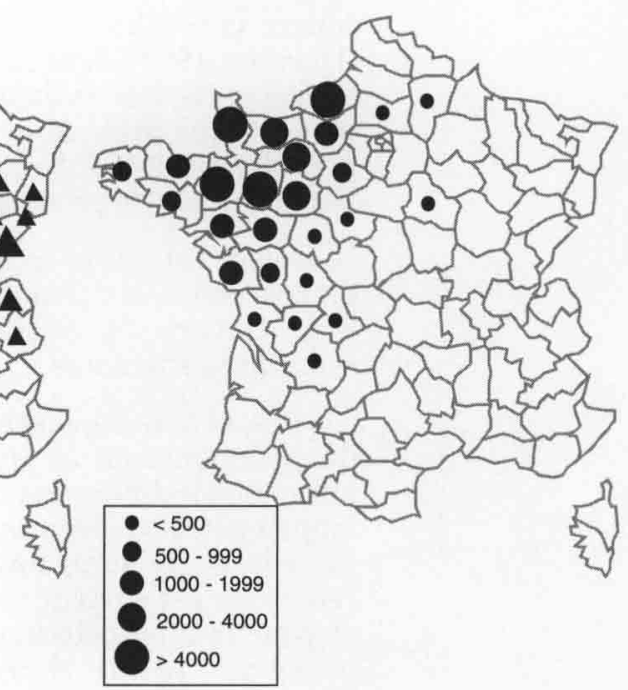

Normande
Normande

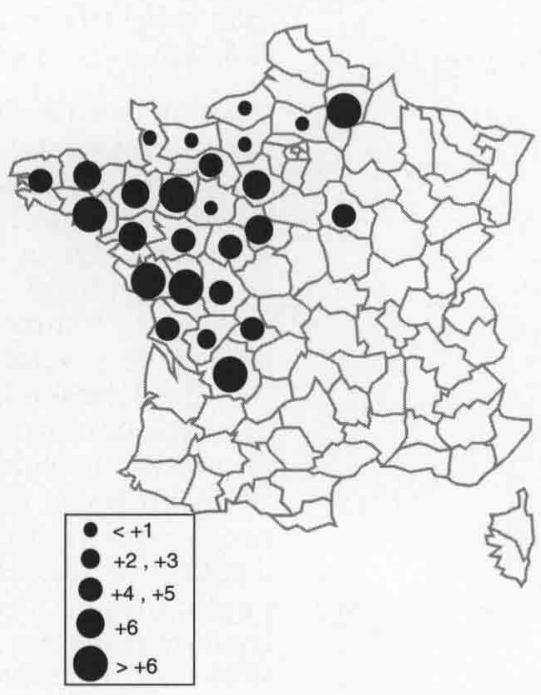

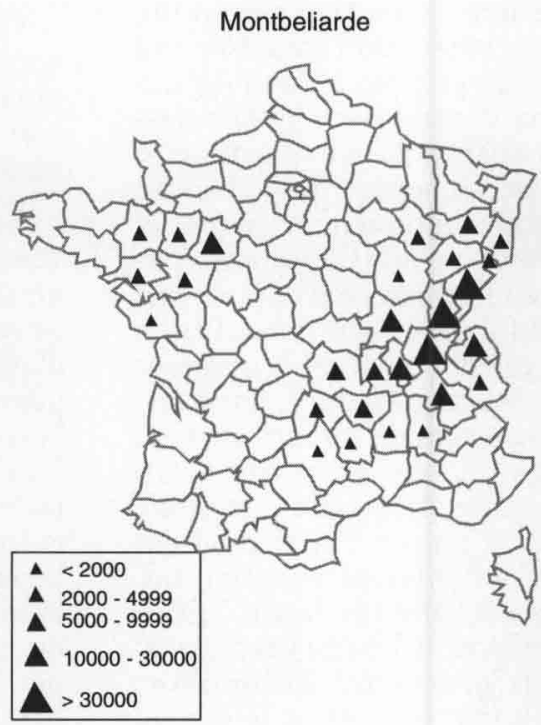

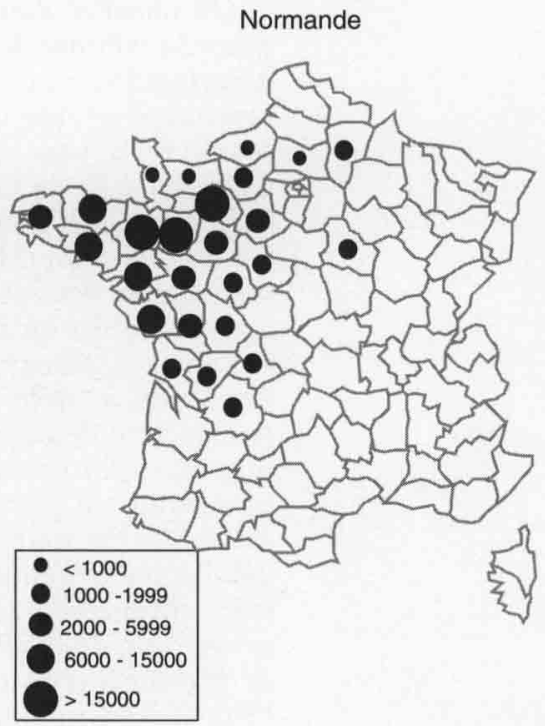


Les résultats relatifs au progrès génétique réalisé ont été largement présentés par ailleurs à l'échelon national (Boichard $e t$ al 1993) ; ils passent souvent sous silence les évolutions indirectes d'autres caractères comme la fertilité et la durée de vie productive (Ducrocq 1993) dont la diminution conduit à exploiter les vaches dans une phase à la fois de plus en plus juvénile de leur vie et de plus en plus défavorable à l'expression de leur potentiel génétique de production laitière.

L'observation de la répartition territoriale de ces nouvelles populations raciales, selon leur critère de désignation, appelle les remarques suivantes :

- les niveaux génétiques augmentent des départements où se trouvent concentrés les anciens sélectionneurs qui ont freiné le développement de l'insémination vers ceux, périphériques de leur zone d'extension. Cette remarque est surtout vraie pour la Normande et pour la Montbéliarde, encore convient-il de noter que la Pie Noire est d'implantation récente en France et que la remarque précédente devrait prendre en compte son noyau originel néerlandais dont l'opposition aux schémas collectifs et aux introductions de gènes Holstein a été longtemps tenace.

- par contre, l'examen des effectifs de la base de sélection (A) montre que les races sont concurrencées sur les marges de leur zone d'extension ancienne : la Montbéliarde et la Normande par la Pie Noire, cette dernière par la céréaliculture (Bassin Parisien), qui apparaît ainsi comme le point d'aboutissement majeur de l'évolution moderniste de l'agriculture dès lors que la qualité des sols et la taille des exploitations s'y prêtent. Il apparaît ainsi de nouveaux pôles de développement génétique, en zones périphériques d'extension des races qui sont communs à plusieurs d'entr'elles, sous l'impulsion notamment des implantations d'entreprises de collecte et de transformation laitière (Plet 1991). Les «Marches de l'Ouest» et, à un degré moindre, le Bassin Lyonnais en constituent les exemples les plus marquants.

On observe donc une opposition apparente entre le volume de la population contrôlée qui détermine la marge de progrès à venir et son niveau génétique qui dépend des effectifs passés de cette base, à égalité de configuration et d'efficacité de la mise en oeuvre des schémas. Cela conduit à proposer la notion de «potentiel génétique" d'un schéma racial (C) sur un territoire (département en l'occurrence) et pour une année donnée en multipliant son effectif utile (A) par son niveau génétique (B) et à suggérer son étude, à travers ses composantes, dans une perspective de prise en compte du territoire en matière d'élevage et de sélection. On remarque, en particulier, le niveau génétique élevé des Pie noires du Bassin Parisien où ont débuté les premiers schémas de sélection laitière intégrée mais où l'élevage bovin laitier est en voie de disparition. A l'autre extrême, si la Monbéliarde est peu représentée dans l'Ouest, son implantation récente et le niveau génétique de sa base de sélection lui confêrent les caractéristiques d'un véritable "front pionnier».

La petite échelle et l'insuffisance de certaines de ces représentations font ressortir l'écart existant avec d'autres indicateurs utilisées classiquement pour traiter de phénomènes liés au territoire : le paysage par exemple si on veut étudier les rapports des populations au territoire et relier les dynamiques observées à des transformations du territoire comme l'établissement des périmètres de drainage. Les travaux non publiés conduits par Sens, en Franche-Comté, à l'échelle des codes postaux qui servent à identifier les codes informatiques des éleveurs, suscitent l'intérêt pour des approfondissements à cette échelle. On objectera, sans doute, que les différences génétiques entre élevages (Agabriel et al 1993) apparaissent faibles dans un échantillon réalisé pour tester des différences de nature des aliments. Mais ceci n'est pas, en fait, sans rapport avec les règles de constitution de tels échantillons par les chercheurs.

\section{2 / Les grandes lignes de la modernisation de l'élevage et des populations bovines}

L'inscription territoriale des populations bovines laitières et ses évolutions depuis deux siècles (soit une trentaine de générations bovines) peuvent donc exprimer l'évolution des systèmes agraires qui leur sont associés sous l'effet des techniques et des choix politiques (Bonnemaire et Vissac 1986, Vissac et Bonnemaire 1987). Les évolutions constatées ci-dessus, qui rejoignent souvent des observations de bon sens, conduisent à noter certaines régularités.

\section{1 / Un élargissement des échelles spatiales d'organisation et un accroissement de la maîtrise et de la spécialisation}

On assiste, parallèlement à l'émergence de nouvelles gammes de populations bovines, à plusieurs types de phénomènes concernant leur gestion et l'organisation de leurs systèmes agraires.

Le volume du cheptel qui détermine de nouveaux types d'opérations collectives s'accroît ainsi que sa diffusion géographique, sous des formes régulières quand il s'agit d'échanges d'animaux, sous des formes plus irrégulières quand elle concerne la semence. A cet égard, si l'expansion des races à partir des populations locales est géographiquement très marquée, celle des souches qui émergent de schémas de sélection pie noirs correspond à un «mitage» territorial. Elle dépend surtout du niveau de maîtrise du milieu, et du bon fonctionnement du marketing des souches, les adaptations climatiques mises à part à un niveau géographique élargi. 
Les objectifs de sélection sont progressivement réduits en nombre jusqu'à n'atteindre pratiquement qu'un seul caractère, les correctifs apportés visant essentiellement à éviter une réduction ou une trop grande dégradation des autres caractères héritables. Cette évolution peut susciter la sélection de formes d'adaptation particulières aux franges de l'écologie des souches. La production est, elle, de plus en plus régulée, face aux risques climatiques et commerciaux, à des niveaux d'organisation élargis : Etat, Communauté (prix de référence, quotas).

La maîtrise de la reproduction et des accouplements est accrue dans chaque troupeau et en référence à l'organisation collective. Cette tendance limite progressivement l'action des individus au profit de décisions d'ensemble qui les contraignent. Si la confusion des «fonctions de juge et de marchand" dominait la scène raciale, l'objectivation et l'apparence démocratique initiale des schémas collectifs ne s'oppose pas à terme à une stratification sociale des éleveurs. Les progrès de la dernière décennie en matière de maitrise de la voie femelle de la sélection peuvent néanmoins stimuler les initiatives d'individus et surtout de petits groupes.

Ces évolutions des populations bovines et des systèmes agraires sont schématisées sur la figure 3 en fonction de leur stade d'émergence. D'une façon générale, alors que la taille et l'assise territoriale des populations augmentent, leurs objectifs et milieux de sélection se restreignent, grâce à la maîtrise technique. Une situation proche, au départ, du milieu naturel fait place peu à peu à des formes de plus en plus accentuées d'anthropisation.

\section{2 / Une accumulation des types de populations bovines par strate et selon des rythmes variables}

L'évolution a entraîné, sur le territoire, le remplacement des populations les plus anciennes par les plus récentes au rythme de l'évolution technologique assimilable par le système agraire. Ces remplacements ont été progressifs et se sont heurtés à des résistances susceptibles de faciliter des adaptations d'usage de populations déclassées.

Dans les situations cumulant toutes les contraintes : insularité, relief, aridité (Corse), les populations originelles se sont maintenues, sans risque d'assimilation, à travers des systèmes hyper-extensifs dégradés. A un degré moindre, les races laitières de montagne se sont maintenues quand elles étaient protégées par des rentes culturelles liées à des produits (Alpes du Nord). Certaines n'ont pu résister qu'en changeant d'orientation : passage de la traite à l'allaitement (Salers et Aubrac dans le Massif Central).

Lorsque les transformations du milieu agricole ont été très rapides, comme dans l'Ouest, on a pu écrire que les gammes de populations ou les races changeaient au rythme d'une par quart de siècle et génération humaine. L'image de la Bretonne Pie Noire exploitant, au début du siècle, des landes d'ajoncs et de genêts a fait place à celle de la Normande, à son stade d'expansion territoriale maximum (milieu du siècle), sur des prairies artificielles fertilisées et exploitées par pâturage tournant. Le développement de la Frisonne Pie Noire a correspondu à la phase d'intensification culturale des années 60 et aux possibilités ainsi offertes
L'évolution s'est faite à un rythme et sous une forme variable selon les systèmes agraires.

Figure 3. Gradients d'évolution des caractéristiques des populations selon leur stade d'émergence dans l'évolution.

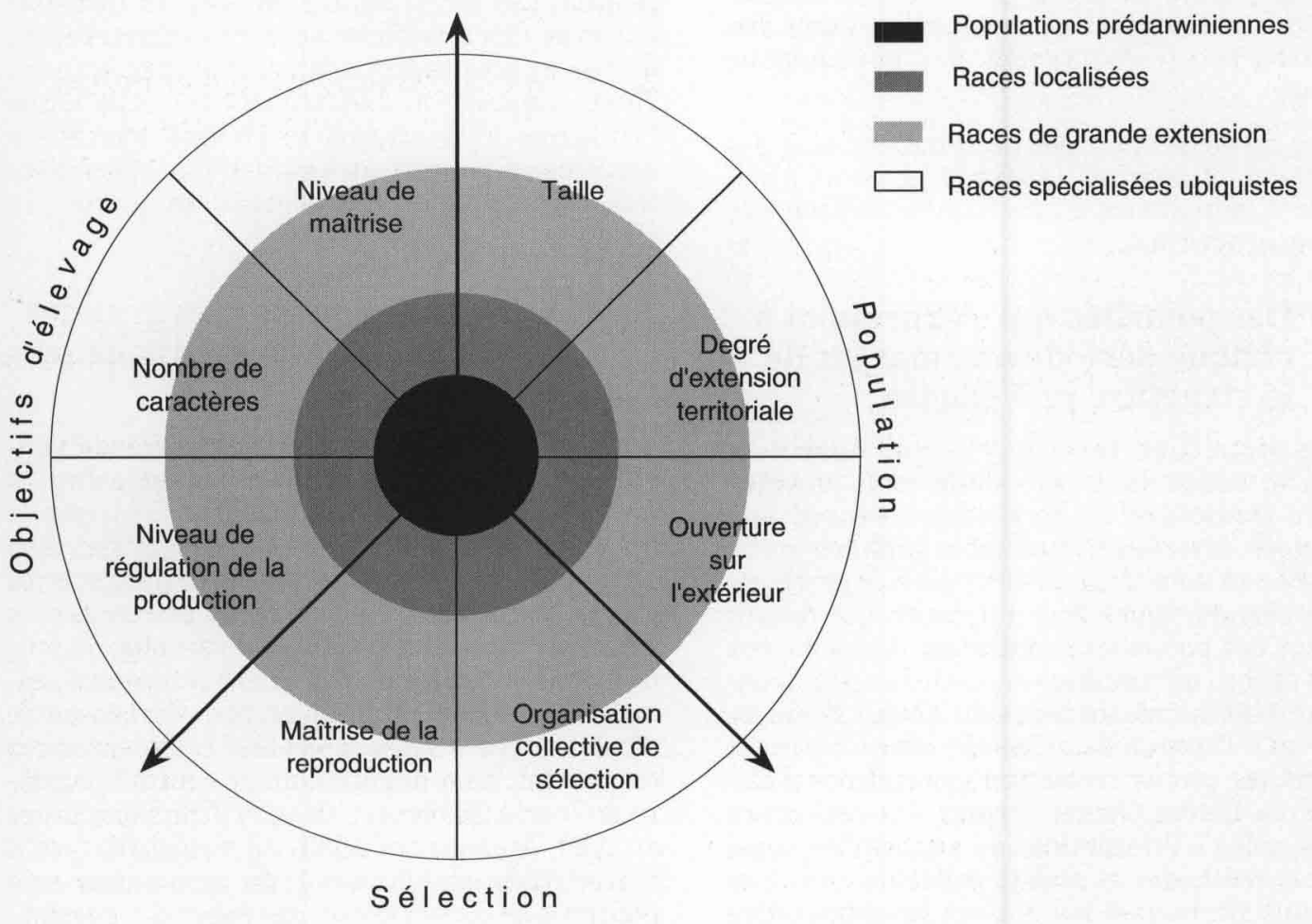


Figure 4. Stratification des systèmes d'élevage et des races en Bretagne.

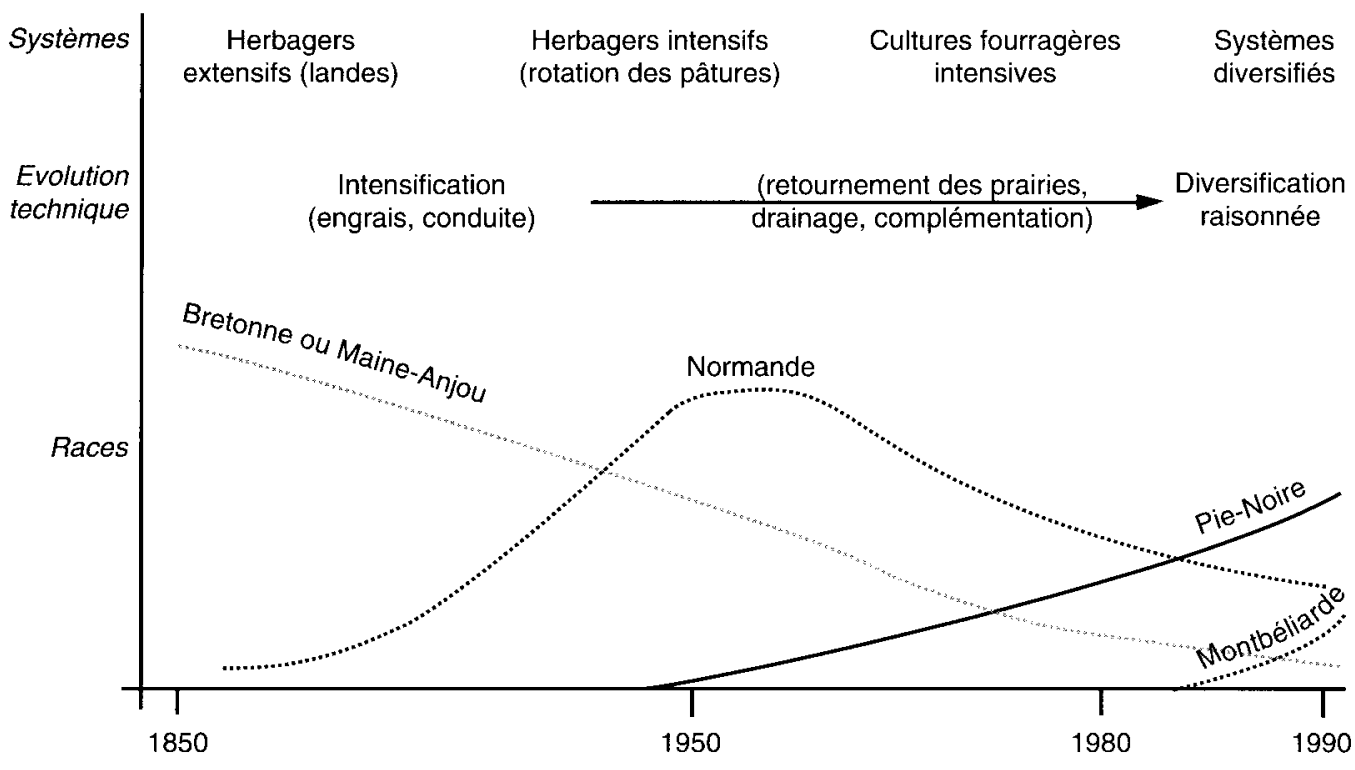

à des éleveurs capables d'intégrer les ressources du progrès technique fourrager sur des sols légers. Il était dans la logique de cette évolution tardive, et appuyée sur d'énormes investissements, d'évoluer vers la holsteinisation seule à même de les rentabiliser.

Ces introductions de nouvelles populations, plus spécialisées pour la production laitière, ne se sont pas soldées par une disparition totale des plus anciennes. La Bretonne pie noire est l'objet d'un programme de conservation cohérent avec les efforts de protection de certaines zones et qui vise à ralentir la perte de gènes de l'échantillon relictuel. Les souches normandes les plus productives se trouvent souvent en Bretagne (figure 2). Lorsque, par contre, l'élevage disparaît au profit de la céréaliculture, cette transformation s'accompagne d'une disparition totale des traces des populations bovines.

Les rythmes et formes du remplacement des populations bovines laitières peuvent ainsi servir d'indicateurs d'une transformation des systèmes agraires.

\section{3 / Des polarités qui s'expriment à chaque période aux marges de la structure précédente}

La structure par "pays" qui dominait jusqu'au début du 19ème siècle était protégée par la fermeture du territoire national aux échanges extérieurs. Les trois grandes races laitières se sont donc développées à partir de pôles périphériques à ce territoire qui constituaient des points de pénétration des gens, des idées et (ou) du matériel animal. Si cette dynamique s'est opérée au cours du 19ème siècle, ce n'est qu'à l'époque de Jules Méline qu'elle s'est concrétisée par la création d'associations d'éleveurs de Livres Généalogiques. La résistance de ces pôles à l'»insémination artificielle", ainsi qu' aux méthodes et enjeux collectifs que cette pratique recouvrait les a alors fait apparaître en négatif par rapport à de nouveaux pôles périphériques aux zones d'expansion raciale sous l'effet des concentrations opérées par l'industrie laitière. Les nouveaux indicateurs (index génotypiques) et la dynamique des populations nouvelles découlent clairement des règles d'une «économie monde» spécialisée.

A chaque phase identifiée par des indicateurs différents, correspond ainsi une structure qui apparaît autour de pôles situés aux marges de la structure précédente (figure 5). La modernisation, dans le domaine de la gestion des populations animales, a donc correspondu à des déterminants techniques qui ont profondément modifié la configuration territoriale et sociale de la mise en oeuvre de cette gestion. Les correspondances avec les transformations des systèmes agraires mériteraient d'être approfondies : au début des années 1980, l'impression dominante était celle d'une tendance à la suppression des systèmes agraires régionaux au profit de la spécialisation laitière et de son vecteur ubiquiste : la Holstein.

\section{4 / D'une approche par les grandes dynamiques à une approche par les résistances}

Ce panorama mécaniste fondé sur les dynamiques dominantes de la modernisation et dont on pressent les limites simplificatrices et socio-économiques doit être complété par une prise en compte des pôles de résistance au changement des populations et des systèmes agraires. Face aux visions conservatoires fondées sur la cryogénie ou des échantillons d'animaux, on se doit de signaler des efforts visant, dés la décennie 1970, à étudier ces résistances en plaçant les animaux dans le contexte agraire de leur utilisation (Vissac 1978, Flamant et al 1989, Audiot et al 1983) : il s'agissait, face à l'incertitude grandissante, de promouvoir une politique de conservation des races qui permet- 
te simultanément d'acquérir une information sur leurs usages et pratiques d'élevage déviants en vue d'aider à la recherche de solutions alternatives. C'est en effet à travers une prise en compte de ces éléments contradictoires qu'on peut réfléchir à des possibilités d'évolution de l'élevage bovin face à la "polycrise», selon Morin et al (1993).

On peut rapprocher plus généralement ces phénomènes d'évolution géographique, anthropologique et biologique des populations animales et des systèmes agraires auxquels elles se rattachent aux phénomènes de mobilité inhérents à l'élevage. Dans ce cadre, on a affaire à des mobilités bioculturelles participant à la définition du système agraire et qui s'expriment sur des échelles, temporelles notamment, de grande ampleur (Vissac 1983). Elles peuvent être interprétées en référence aux travaux de Vavilov (1925) sur l'émergence des foyers de domestication et correspondraient à des foyers secondaires d'innovations à partir desquels Lauvergne (1979) a étudié les modes de migration des populations ovines.

\section{3 / Réflexions prospectives sur la crise de l'élevage}

Ces éléments historiques traduisent donc les limites d'un processus de spécialisation des systèmes agraires et des populations. Au fur et à mesure que ce processus se développe sur la petite échelle géographique et le temps long, il génère, sur les plus grandes, des résistances en forme de rupture. Cette opposition d'échelles d'expression rejoint celle entre un ordre ancien et mécaniste et un désordre récent qui fait penser à un nouveau "MoyenAge" (Minc 1993) : cette expression traduit surtout, en fait, notre incapacité à gérer ce qui paraît être un nouvel ordre imposant d'intégrer, dans les raisonnements sur la gestion de l'élevage, les rapports aux filières de produits et aux territoires aux échelles où se manifestent ces rapports. Comme le souligne Aglietta (1981), "dans les crises, se forment des conjonctures instables dont l'issue n'est pas contenue dans les processus qui ont participé aux régimes de fonctionnement passé du système. Tout se passe comme si le système était contraint à la mutation mais que des bifurcations soient possibles sans que telle ou telle voie soit «assignable».

Ces évolutions contradictoires interviennent alors que des changements techniques sont en passe, en élevage laitier notamment, de supprimer la plupart des barrières qui s'opposent aux mouvements de l'information (temps réel) et du matériel (semences, embryons). Ces changements peuvent aussi faciliter des initiatives à des niveaux plus locaux et conviviaux en liaison avec des systèmes d'élevage adaptés aux besoins des filières et des territoires, sous réserve de disposer des modes de représentation correspondants. C'est loin d'être le cas ; comme le souligne Gruson (1987), rejoignant ainsi les réflexions ci-dessus sur les échelles, on est confronté, là comme ailleurs, au problè-
Figure 5. Polarités successives de développement des populations laitières aux marges des structures organisantes précédentes.

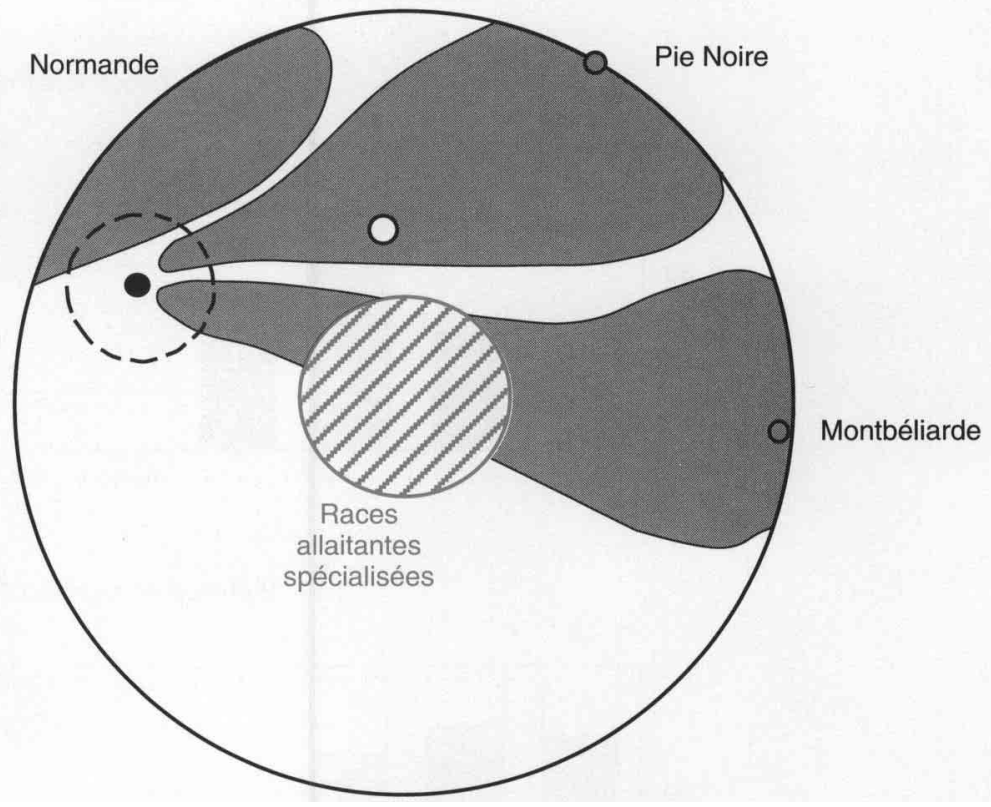

Pôle et périphérie du territoire national $(<1880)$

Pôle et périphérie des territoires raciaux (1950)

Pôle et périphérie des bassins laitiers et schémas liés (2000 ?)

me de l'adaptation des «structures lourdes» à des niveaux de décision inférieurs.

Dans un tel contexte, qui met en cause des modes de raisonnement datant de la Renaissance et du cartésianisme, il semble nécessaire d'élargir le débat et de le situer d'emblée dans le cadre des postulats de globalité et de systémique (Morin et al 1993). Ce dernier s'appuie sur le processus d'»hominisation» en y associant ses composantes bio-écologiques, psychologiques et socio-culturelles en vue d'une recherche d'un "metadéveloppement». Si cette ambition est à la hauteur de l'enjeu, comment peut-on la traduire concrètement au niveau des systèmes agraires et des populations bovines laitières correspondantes? On mesure déjà, à cet égard, l'entorse qui est faite aux postulats précédents, en réduisant le problème à l'élevage laitier et les limites des raisonnements qui en résultent.

A l'instar de Morin et al (1993) nous traiterons séparément des trois composantes ci-dessus dans leurs rapports à l'élevage des bovins laitiers.

\section{1 / Ecologie bovine et systèmes agraires}

Les résultats de la sélection laitière sont donc absolument remarquables (Boichard et al 1992) mais concernent des conditions d'élevage de plus en plus maîtrisées. Pour rester dans la limite des hypothèses de base de la génétique quantitative appliquée, les chercheurs se trouvent, par ailleurs, limités dans 
Figure 6. Relation entre la fertilité et le format des bovins (Massif des Beni Salah, Algérie).

Fréquences des formats des vaches $(\%)$
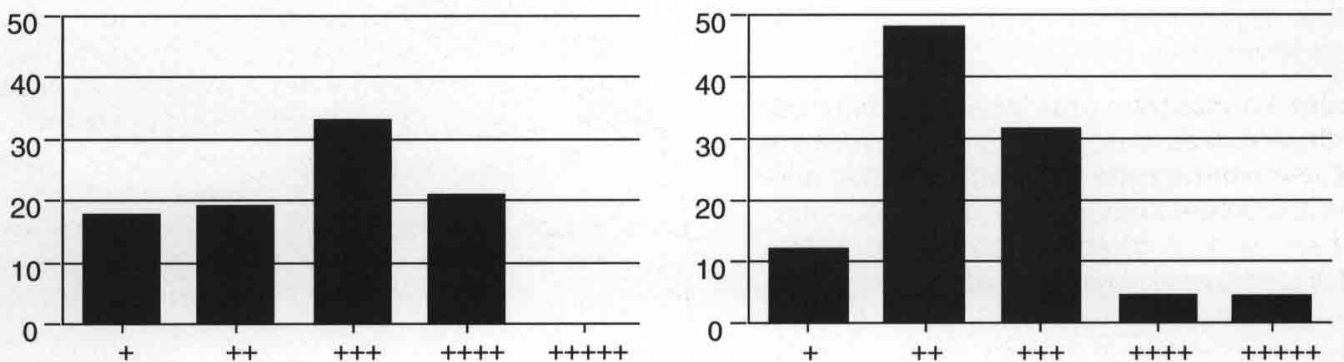

Rythmes de reproduction et formats des vaches (\%)
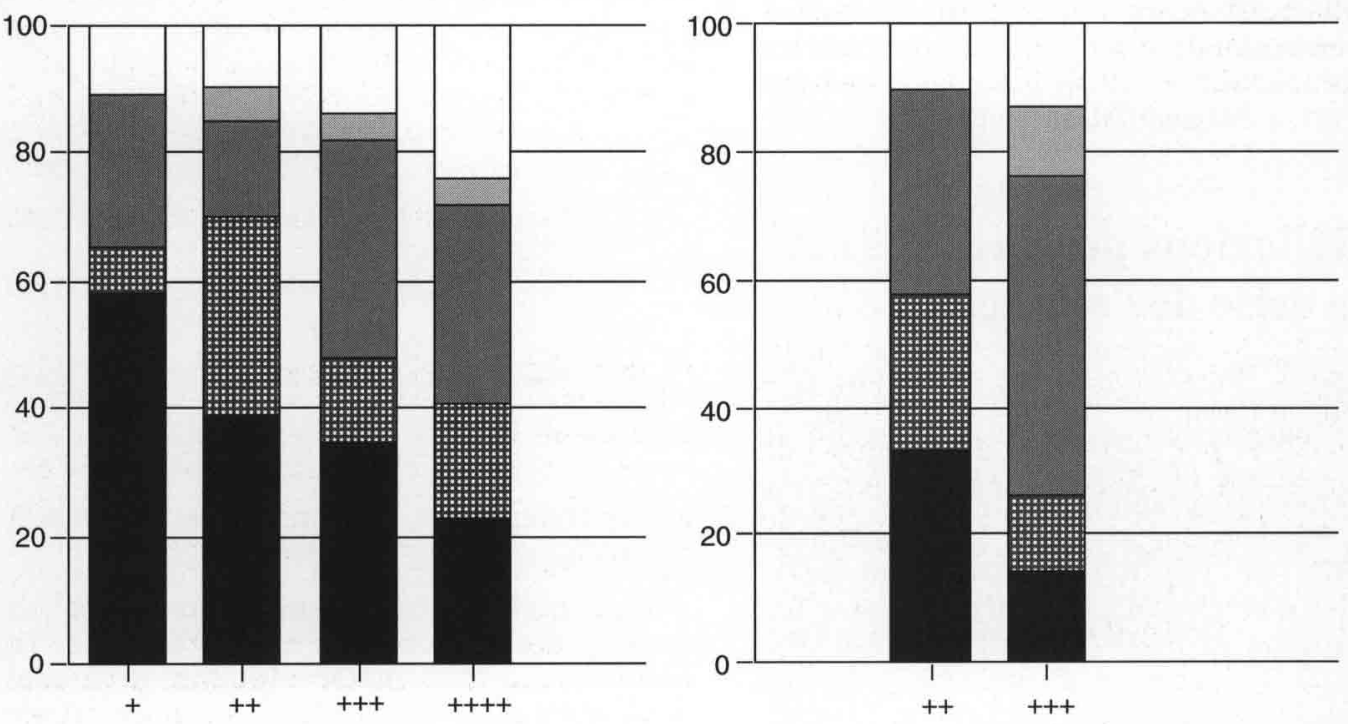

\begin{tabular}{llc}
\hline Vêlage au moins 3 années successives & $\begin{array}{c}+++-+ \\
+++++\end{array}$ \\
\hline Vêlage 2 années successives et jamais 2 manques successifs & ++-++ \\
\hline$\square$ & +++-+ \\
\hline$\square$ & Vêlage une année sur deux 2 années successives suivies de 2 ans de "repos" & +-++ \\
& ++-+ \\
\hline$\square$ & Vêlage une année sur trois au plus & +--+- \\
\end{tabular}

les méthodes et validations correspondant à la prise en compte des relations entre plusieurs caractères compte tenu, notamment, des oppositions entre quantité et composition du lait, entre quantité de lait et de viande (Mallard 1992).

Qui plus est, l'impossibilité de sélectionner des caractères peu héritables, mais essentiels à l'expression de la production comme la fertilité, laisse planer un doute sur l'évolution des phénotypes qui déterminent, en définitive, le revenu des éleveurs, si le «milieu» change. La faible héritabilité observée n'est-elle pas liée aux bornes de variation imposées au milieu. On remarque, à ce sujet (Madani, 1993), à partir d'une population bovine algérienne très croisée entre la population locale et des cheptels de races françaises laitières, populations dont les formats varient de 1 à 3 , que la fertilité (séquences de mises-bas annuelles) diminue régulièrement avec l'augmentation du format (figure 6). Si les conditions extrêmes de ce contexte sont heureusement rares chez nous, l'incertitude prégnante exige de s'en prémunir. Cela implique de préciser les limites de l'écologie (au sens des pratiques d'élevage) d'une race ou vice-versa de la sélection des caractères pris 
en compte dans la sélection sur les autres notamment la fertilité, la longévité, pour une écologie donnée. On objectera que la production laitière est devenue l'apanage de situations d'élevage très maîtrisées et doit faire place à d'autres types d'élevage si on s'en écarte : cela ne supprime pas pour autant l'interrogation, au contraire.

Prendre en compte simultanément les incidences de l'élevage sur la production (filières) et sur l'«environnement» implique de considérer simultanément les deux types de rapports existant entre l'élevage et le territoire : ce dernier est le support de l'élevage auquel il procure les ressources alimentaires des animaux (filière) ; à l'inverse, il est aussi le produit de l'élevage à travers les modifications d'aménagement du territoire, les pollutions qu'il génère par excès ou, par défaut, les abandons d'utilisation (environnement). Ces deux types de relations sont évidemment contradictoires quand les systèmes d'élevage évoluent vers des extrêmes d'anthropisation. Mais elles mettent en jeu des échelles territoriales variées et différentes; elles s'expriment sur des pas de temps et des échéances, différentes elles aussi : ceci justifie leur approche par le postulat de complexité.

En ce qui concerne la première relation, l'extension générale des modèles laitiers intensifs et de la Holstein au profit des jeunes éleveurs sur le territoire a généré des délocalisations facilitées par l'extension des schémas de sélection et des pratiques intensives (engrais et aliments du bétail). Les races sont sorties de leurs limites écologiques (le "croissant laitier" en première approximation) ; nombre d'éleveurs ou de successeurs et d'organisations se sont trouvés fragilisés face aux multiples contraintes imposées par les caprices du climat, la disparition des garanties et des régulations communautaires (quotas), la pression des coûts des intrants. L'expression de "resserrement du ciseau des prix" ne résume qu'imparfaitement un syndrome dont les poussées sont convulsives et différemment exprimées, selon les lieux, l'importance et l'ancienneté des investissements.

On peut, pour illustrer cela à l'échelle départementale, se fonder sur les estimations des effets du milieu d'étable sur la production de matières utiles par lactation qui constituent les compléments, par rapport au phénotype, des effets génétiques précédents (figure 7 , homologue de la figure 2 tirée de : ITEB-INRA 1986 à 1988). La première série d'estimations (partie supérieure) correspond aux valeurs moyennes de ces effets non génétiques de 1985 à 1988 pour les étables d'une race et d'un département. La deuxième série (partie inférieure) représente l'inverse de l'écart-type des moyennes annuelles et traduit notamment la stabilité de ces moyennes selon les changements climatiques et les parades que les éleveurs peuvent leur apporter par le biais de leurs pratiques.

On observe une remarquable concordance d'ensemble des deux cartes d'une même race et des zones écologiques "géographiques" qu'elles traduisent. Des différences apparaissent néanmoins : ainsi, la stabilité interannuelle est relativement meilleure en Pie Noire dans le Pays Basque, la Lorraine et l'Alsace ; elle est plus médiocre dans le Nord Picardie. Il ne s'agit là que d'une illustration globale liée à quatre années. Elle ne peut prendre un sens opératoire qu'à des échelles plus locales : les travaux engagés en Franche Comté sur la race Montbéliarde, à une échelle se rapprochant des communes : code postal (Sens, non publié), se situaient dans cette voie. Plus précisément, pour avoir un sens zootechnique, ces estimations des effets du milieu d'élevage devraient être confrontées à des pratiques ou combinaisons de pratiques d'élevage. Les procédures élaborées par Perrot (1991) en Haute-Marne sont, à cet égard, adaptées à la constitution d'une base de données susceptible de faire le pendant de la base réalisée par les généticiens et d'en étendre l'utilisation à des fins d'aide au développement. Il reste qu'on est loin du compte ; les travaux utilisant de tels rapprochements (Agabriel et al 1993) restent en effet limités à la constitution d'échantillons visant à comparer des natures d'aliments et à étendre le champ d'application des connaissances sur l'alimentation des animaux. Ils mériteraient d'être étendus dans cette voie.

Que le territoire soit le reflet de la race et de son mode d'élevage ressort des contrastes entre les paysages normands, franc comtois et briards où étaient implantées les trois races dans la première moitié du siècle. Si ces contrastes se sont largement estompés sous l'influence des pratiques d'aménagement agricole (remembrement, drainage, arasement des haies), le paysage peut constituer un outil de caractérisation des systèmes agraires utilisable à des fins de développement rural (Deffontaines 1972). Il peut être rapporté à des indicateurs particuliers de qualité de l'environnement, comme par exemple la teneur des eaux souterraines en nitrates, l'intensité de l'érosion au niveau d'un ensemble territorial cohérent avec les phénomènes en cause. Pour prendre en compte l'incidence de l'élevage à ce niveau, il faudrait considérer simultanément les conséquences des interventions réalisées sur les lambeaux de territoire que constituent les ensembles de parcelles des exploitations qui l'occupent (Benoit 1993). Ainsi, dans le cas des pollutions par les nitrates, on doit s'intéresser à des aspects aussi divers que la taille des troupeaux, la concentration des "pissats" autour des points d'abreuvement, celle des épandages sur des parcelles particulières hors assolement.

Devant une telle diversité d'éléments on est alors conduit à envisager globalement les rapports existants entre les races, leurs modes d'élevage et, plus largement, les systèmes agraires et les paysages qui les caractérisent. Un système de pratiques, Cristofini et al (1978), associant l'arasement des haies, la culture du maïs fourrage, le zéro pâturage, l'ensilage et la concentration de l'épandage des fumiers sur telle ou telle parcelle n'est-il pas mis en oeuvre préférentiellement par des éleveurs de Holstein plutôt que par ceux de montbéliardes
De la sélection génotypique au phénotype de «l'animal entier élevé»dans son système agraire 
Figure 7. Effets du milieu par département et par race (1985 - 1986 - 1987 - 1988). Les données de base sont les effets des élevages d'un département pour chacune des 4 années et des 3 races, après correction des effets génétiques, de l'âge des femelles notamment. Ils sont exprimés en déviation par rapport à une moyenne nulle. L'effet moyen est la moyenne des 4 déviations annuelles. La stabilité est exprimée en classant dans l'ordre inverse les valeurs de l'écart-type des déviations entre les 4 années.

Pie-Noire

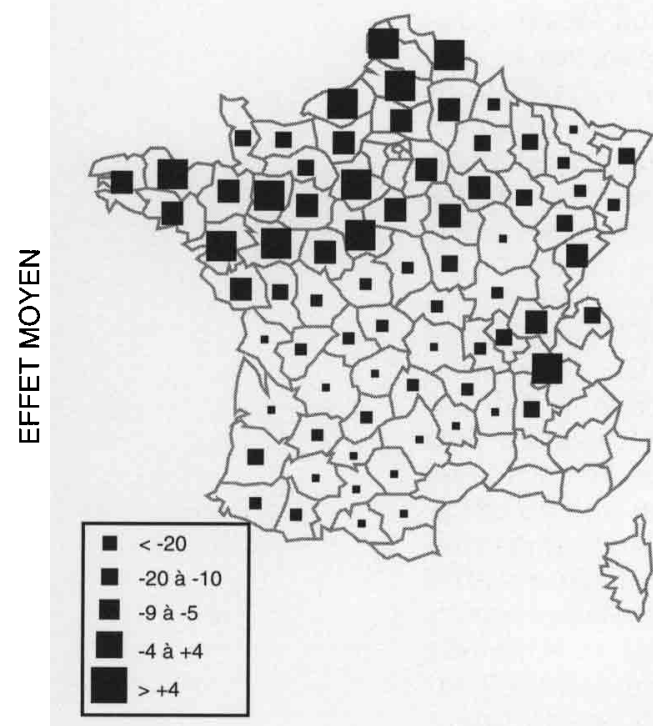

Montbeliarde

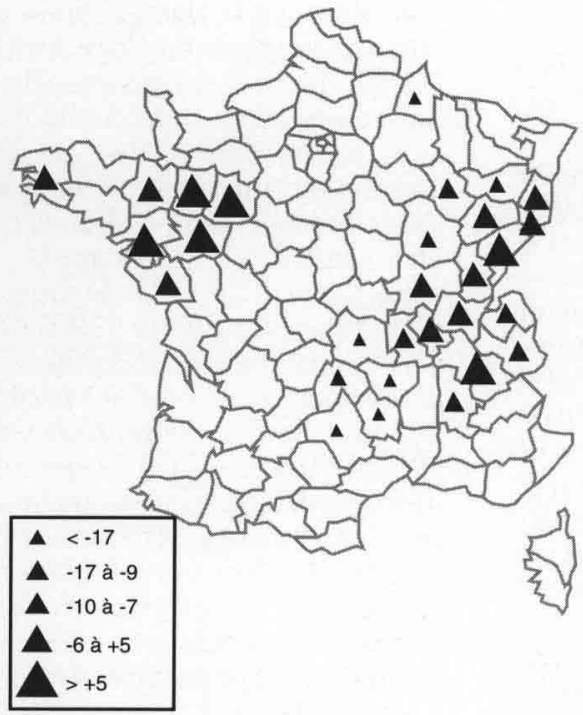

Normande

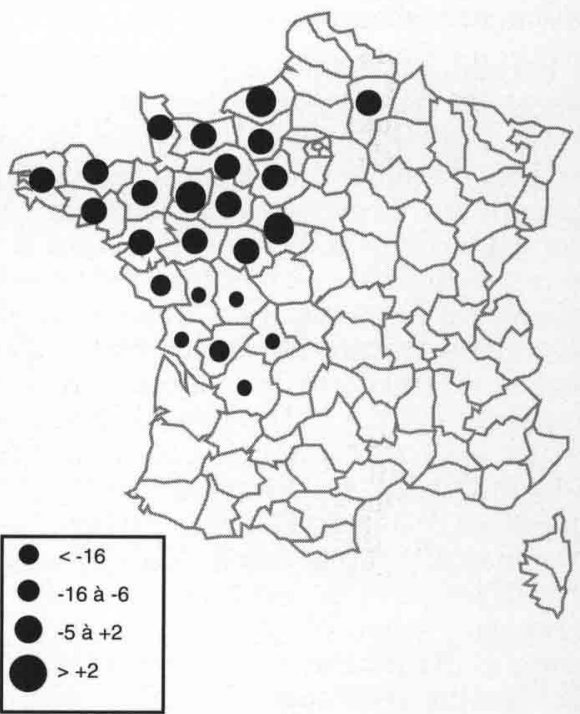

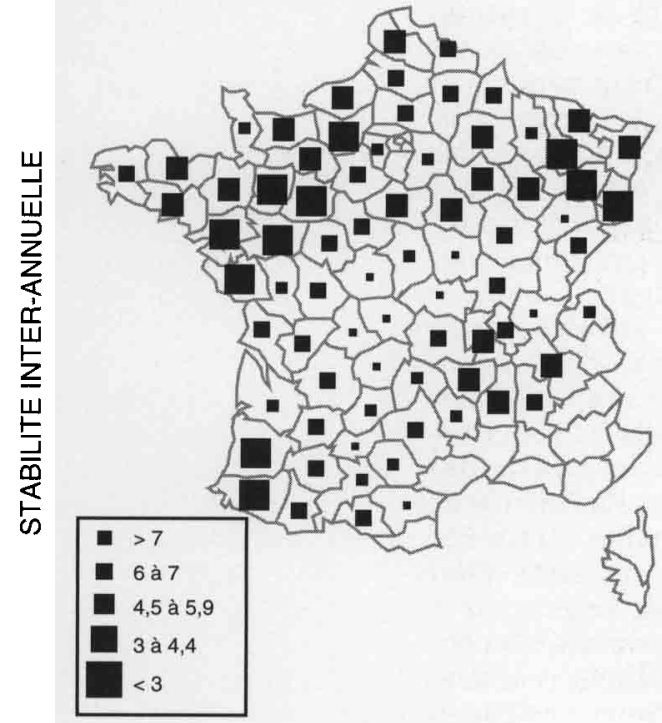

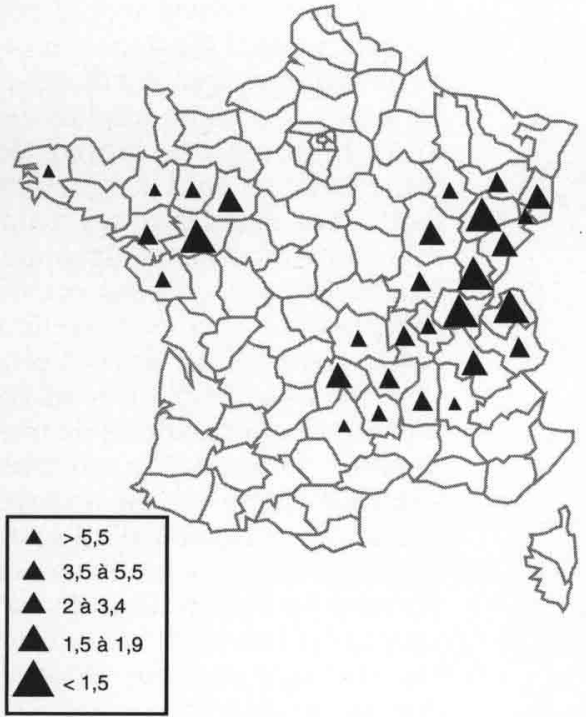

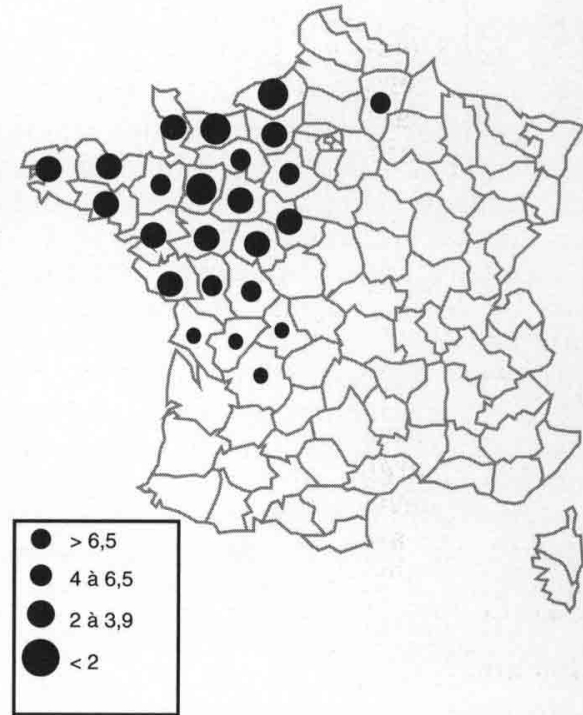

et de normandes ? Changer un tel système de pratiques n'est-il pas plus pénalisant pour les premiers ? Bref, la race animale et le cortège de pratiques auxquelles elle est soumise ne sont-ils pas à prendre en compte avec d'autres indicateurs particuliers pour trouver des voies plus raisonnables de gestion des territoires ruraux où domine l'élevage laitier?

\section{2 / Complexité, incertitude et décision en élevage}

Il y a loin ensuite du diagnostic à la décision. Les éleveurs se trouvent en effet confrontés à une situation qui heurte leurs stratégies de développement : réduction de taille du troupeau laitier, adjonction d'un troupeau d'allaitantes, incitations à la recherche de qualité des produits et pressions diverses des collec- teurs de lait selon leur situation par rapport aux exploitations. A cela s'ajoutent les exigences «environnementales" manifestées par la société locale : ces exigences sont d'autant plus fortes qu'ils sont devenus plus minoritaires. Ce qu'on appelle l'environnement ne se traduit pas en effet, comme on peut avoir tendance à le penser, par des normes établies ; en Lorraine, face au déferlement de la "vague verte", venue de l'Est, les exigences écologiques sont plus fortes qu'en Bretagne où le poids de l'agro-alimentaire est dominant dans les stratégies politiques locales. Ainsi la notion de qualité, qu'elle concerne les produits ou l'environnement de l'élevage, doit être considérée comme liée à une base sociale régionale qui la construit ; elle se réfêre ainsi directement au système agraire. 
A la complexité de cette situation s'ajoute une incertitude sur l'avenir qui est d'autant plus grande que, selon l'expression de Morin et al (1993), la relation passée/présent/futur dans les représentations (des éleveurs laitiers) se trouve "desséchée, atrophiée ou bloquée». Ces derniers sont souvent placés, en effet, devant un double interdit culturel : au rejet de leurs racines à travers une race insuffisamment productive (Salers, Aubrac) et (ou) porteuse d'une image rétrograde dans leur région (Normande dans l'Ouest) s'ajoutent les critiques contre le «productivisme» et les inconvénients qualitatifs de la Holstein qu'on leur a pourtant érigée en modèle pédagogique au lycée. Certains, devant ce dilemme, vont chercher ailleurs une race différente sur la base de justifications partiellement objectives. La Fleckvieh Bavaroise constitue, pour les Aubraciens, un palliatif leur rappelant les laits de leur race insuffisamment productive et leur évitant le recours à la Pie Noire. Les promoteurs de la Montbéliarde dans l'Ouest lui trouvent une parenté de couleur de pelage avec la MaineAnjou mais des caractéristiques laitières et bouchères globalement supérieures aux siennes et à celles des autres races laitières utilisées dans la région.

Rares sont en effet les situations agraires qui se prêtent à l'utilisation d'un cheptel mixte pour que les éleveurs puissent faire face eux mêmes, aux dérégulations des marchés et aux aléas divers, sans recourir, dans leur exploitation, à la conjonction des extrêmes de la gamme raciale (troupeau laitier Holstein et troupeau allaitant charolais) ou à recourir au croisement viande sur leur troupeau laitier. $\mathrm{Ce}$ dernier est, en effet, difficile à maîtriser sur des troupeaux de petit effectif. L'exemple de la Pie Rouge de l'Est en Côte d'Or serait particulièrement intéressant à cet égard pour l'étude d'un modèle racial alternatif : là, en bordure du Bassigny argileux, herbager, et du Barrois céréalier se développent des systèmes d'élevage autonomes associant ces deux types de territoires et exploitant la mixité de la race Pie Rouge de l'Est à travers des combinaisons autonomes des deux types de troupeaux (laitier et allaitant), du naissage et de l'engraissement. Faire face soi même à l'incertitude peut être réalisé en disposant conjointement de types raciaux et de ressources alternatifs pour pouvoir augmenter le «jeu des possibles» face à l'incertitude ambiante.

Prendre en compte ce jeu des acteurs désorientés est devenu d'autant plus important que, comme on l'a précisé, les facilités de diffusion des informations et des nouveaux vecteurs de la sélection accroissent les possibilités de choix... et d'erreurs. Les délocalisations de races par rapport à leur terroir d'origine, qui vont résulter de cette situation, peuvent porter en germe des innovations si elles sont étudiées dans leurs rapports au système agraire. Mais ces phénomènes de grande ampleur spatiale et temporelle conduisent aussi à attirer l'attention sur le risque qu'il y aurait à dissocier dans la gestion à long terme des populations : le biologique, supposé affaire de cryogénie, et le culturel, supposé affaire de muséologie. La dispa- rition du lien humain et social entre les deux ne permet pas en effet ou très difficilement de reconstituer des combinaisons opératoires.

\section{3 / A la recherche de nouveaux liens socio-culturels}

Envisager l'élevage bovin dans sa dimension culturelle à des fins d'aide au développement est une gageure bien difficile pour le chercheur agronome sur qui la société a fait reposer une confiance sans limites dans la fourniture de «solutions» conformes aux enjeux de son agriculture.

Cela met d'abord en cause son éthique de chercheur et ses paradigmes traditionnels : contrairement au contexte de la loi sur l'élevage, dite loi génétique (Pluvinage), les questions ne sont pas formulées simplement, elles émanent des acteurs et sont en général conflictuelles ; les règles de solutionnement dans le «complexe» échappent au principe classique d'optimisation (Poutous et Vissac 1962) pour rejoindre des formes dialectiques procédurales. Le chercheur n'est pas indépendant du contexte (principe du tiers exclus) dans la recherche d'un modèle objet, il en est partie prenante (principe du tiers inclus) et le processus de Recherche-Action vise à aider les éléments de la société à réaliser des arbitrages successifs entre des exigences apparemment contradictoires dans leur formulation initiale. Ce retournement dialectique de la position du chercheur («problem finding to problem solving") est une situation noble mais difficile à vivre pour des chercheurs responsables de la gestion des bases de données ("problem solving»).

Il convient d'abord de comprendre la situation de l'élevage caractérisée par un jeu d'acteurs multiples interagissant. Cela va bien au delà du jeu des débats professionnels qui présidait à la conception et à l'élaboration de la loi sur l'élevage. Les acteurs en cause aujourd'hui entretiennent des relations complexes à des échelles variables. Ce sont, bien entendu, les éleveurs et leur infrastructure d'encadrement, leurs partenaires de l'agroindustrie qui pèsent sur cette dernière en amont (intérêt des firmes industrielles pour les biotechnologies) et en aval (transformation des produits), les aménageurs et gestionnaires des espaces ruraux et, au bout du compte, les consommateurs de produits, d'espace, d'eau qui sont les cibles de risques multiples (notion de coproduits de l'élevage).

La conjonction de ces parties prenantes s'opère à travers des canaux sociaux et territoriaux très divers dans leur nature, leur structuration, leurs règles et leur extension : des techniciens locaux aux organismes départementaux qui encadrent l'élevage jusqu'aux instances régionales et nationales, des "bassins laitiers" aux grands groupes industriels, des bassins versants aux agences de bassin par exemple...Ces niveaux et ces échelles d'organisation s'entre croisent entre structures selon de véritables systèmes de pratiques humaines collectives répondant à des objectifs et obéis- 
sant à des logiques différentes qui participent à la construction sociale locale, régionale, nationale (Vissac 1989). Les différences s'expriment souvent en terme d'échéances et de lieux sensibles. Ainsi, par exemple, l'élevage a des exigences souvent immédiates là où il est mis en oeuvre (ici et maintenant), des conséquences différées (ici et plus tard) pour la conservation des races ou plus lointaines (ailleurs et plus tard) en matière de pollutions de nappes et d'érosion des sols. Ces catégories d'analyses sont reprises de Legay (1993).

Les échelles intermédiaires apparaissent intuitivement les plus adaptées à la prise en compte des phénomènes culturels intéressant les filières de produits et les territoires en rapport avec les populations animales.

Le cas de l'Aubrac fait à cet égard figure de cas d'école : une race associée à un établissement humain très localisé mais qui présente néanmoins l'inconvénient d'être écartelé entre trois régions de programme. Ce n'est d'ailleurs pas nouveau : la Croix des Trois Evêques à Saint Urcize n'était-elle pas, depuis des lustres, le point de rencontre des hautes hiérarchies ecclésiastiques de Mende, Rodez et Saint Flour appartenant à trois départements, à trois provinces, réseaux hydrographiques et... régions de programme aujourd'hui ? Cette race et son établissement humain, fondés sur la transhumance estivale des troupeaux de vaches, ont connu des évolutions rapides qui se sont superposées au cours du dernier demisiècle (Rouquette 1993). L'"esprit des lieux" traduisait le mode d'exploitation de la race avant la guerre de 1939-1945; l'équipe de buronniers engagée pendant l'été pour traire et fabriquer dans les burons la fourme de Laguiole gagnait ensuite la capitale pour travailler pendant l'hiver dans les commerces des locaux émigrés ; la famille et les relations locales étaient à la base de ce système de solidarité tant en matière de travail que de financement. Mais, le coût et les astreintes sociales de ce système, face à la faible productivité laitière des vaches, ont alors conduit les éleveurs, dés 1962 et sous l'impulsion de l'INRA dans le cadre de la RCP Aubrac (Vissac 1968), à développer une forme d'élevage allaitant combinant en croisement les aptitudes maternelles de la femelle Aubrac avec le potentiel de croissance musculaire des taureaux charolais : c'était, pour les éleveurs, l'invasion de l'»esprit du ranching américain* qui a fait la fortune de la race dans quelques régions avoisinantes. Mais, certains éleveurs, préoccupés du maintien de la fabrication du Laguiole auquel était associé l'Aubrac et insatisfaits des laits de Pie Noire, se sont alors tournés vers des introductions de Fleckvieh bavaroises et de sa "cousine", la Pie Rouge de l'Est, dont les laits étaient de meilleure qualité. A travers ce qu'ils appellent le retour à l'»esprit de la vieille Europe» ils ont imposé un cahier des charges très dissuasif pour les pies noires et ils ont remis en valeur un fromage de qualité et de longue conservation. Enfin, dans l'ambiance des quotas laitiers et de la promotion de la qualité, le «fer de lance» de ces derniers envisage même un retour à l'«esprit des lieux» en conservant les meilleures souches laitières d'Aubrac dont la multiplication est devenue techniquement possible grâce au clonage et au transfert des embryons. Cette dynamique, qui voit se superposer des références associant différemment la culture introduite et la culture locale, se traduit par des débats internes mais aboutit présentement à une situation de valorisation des produits «haut de gamme» de la race et de son terroir (fourme de "vieille garde», croisés charolais dénommés "fleurs d'Aubrac", coutellerie locale et surtout gastronomie naturelle valorisant les «herbes» du terroir).

La situation de la Tarine est sensiblement différente : son statut local, dans le Beaufort, associe une valorisation protégée par le biais d'un fromage "haut de gamme" avec le besoin d'entretien par le pâturage de surfaces pentues du "tourisme blanc» de grand renom; elle est la seule race laitière de ce niveau de production à pouvoir assurer cette deuxième fonction dont la réalisation est hypothéquée par la tendance à l'élevage à l'auge à base de foin de Crau. A cette version emblématique de la race, dans son noyau originel, s'ajoutent des dérives liées à son expansion dans les zones méditerranéennes suivant des formes associant la traite et l'allaitement (voir plus haut, Madani 1993) ; plus récemment, les qualités maternelles de la Tarine ont été révélées expérimentalement (USA), en croisement avec l'Áberdeen Angus, en vue d'un deuxième croisement avec des taureaux à viande de races à forte musculature. Cette diversité d'usages et de pratiques d'élevage constitue un potentiel à mieux connaître et exploiter pour la sélection à travers un contrôle conjoint des performances et des pratiques. Les effectifs réduits de la Tarine dans les Alpes impose, à l'évidence, une telle vision élargie de sa gestion.

Dans le cas des races de grande extension, on ne trouve pas, comme précédemment, de correspondance simple entre une race et un territoire. C'est, en général, la pression locale qui peut imposer des adaptations à des situations pluriraciales. Elle peut provenir d'un besoin d'adaptation de la matière première aux impératifs de la transformation et de nécessités imposées par la gestion de zones sensibles du territoire dans une perspective de relations entre les races et les systèmes d'élevage (voir ci-dessus). Sur le premier aspect, on peut citer le cas de la vallée d'Aspe. En dehors de ses ours et problèmes de jonction transpyrénéenne, elle dispose encore de troupeaux bovins pluriethniques où la Blonde des Pyrénées est maintenue pour compenser les inconvénients de fromageabilité des laits des pies noires (Bertocchio 1989) en vue de produire le dernier fromage mixte : vache-brebis connu en France, à notre connaissance. C'est surtout, chez les bovins, au niveau des laiteries, que sont soulevés les problèmes les plus aigus de qualité des laits : les critiques formulées à ce sujet sur les orientations des schémas de sélection ne concernaient-elles pas surtout, en fait, une conséquence locale de la transformation progressive des cheptels normands ou montbéliards d'éleveurs âgés par des cheptels pies noirs de nouveaux installés (Gilibert et 
Courbon 1990) ? Sur ce deuxième aspect, si des palliatifs d'urgence doivent être trouvés dans certaines situations à grand risque (Vittel) : limitation de la surface en mais au profit de la luzerne, compostage des déchets (Benoit, 1993), le problème du choix racial et de sa valorisation par les industriels n'est-il pas posé plus largement? Derrière des exhortations trop générales sur ce problème (plans de développement durables), on a affaire, en définitive, à un besoin d'intégration des incitations provenant des industries laitières sur la qualité des laits (filières) et des agences de bassin (territoires) qui prenne en compte les besoins du marché des produits et les réactions sociales aux effets environnementaux des modèles d'élevage, dans des situations définies et non en termes généraux.

En dehors des critiques d'échelles où se posent ces problèmes, critiques qui concernent une application totale du principe de «subsidiarité", la difficulté majeure de mise en oeuvre de telles mesures globales ne réside-telle pas dans la différence de logiques sous jacentes : le marché dans un cas (fillière), la collectivité publique dans l'autre (territoire) ? L'économie des conventions semble offrir quelques pistes intéressantes pour dépasser ces contradictions de logiques.

Il est donc envisageable, se référant à la modélisation systémique, de traiter du problème des rapports de l'élevage bovin laitier aux filières et aux territoires à des fins de gestion globale de l'élevage bovin laitier et de considérer la population animale comme un guide bioculturel du développement rural. Cela impose un changement des modes de raisonnement et de l'éthique des chercheurs : ils doivent intégrer leur culture dans les modèles et appuyer leurs interventions sur une connaissance plus complète et rigoureuse des représentations et pratiques des acteurs et des consommateurs dans leurs rapports au territoire et aux produits de l'élevage.

\section{Conclusion}

La référence à l'histoire des populations domestiquées en rapport avec celle de leurs systèmes agraires est, en définitive, essentielle à qui veut envisager actuellement la maîtrise de leur évolution. On peut, en effet, s'étonner avec Legay (1993) de l'insistance des chercheurs, dans l'engouement des biotechnologies, à explorer les origines de la domestication et les voies récentes de manipulation du génome sans avoir la prudence d'explorer la longue "phase intermédiaire" où se sont manifestés les rapports de l'homme au matériel vivant.

L'observation du matériel animal et des systèmes d'élevage bovin laitier, jugés représentatifs de la modernité, traduit donc une évolution vers la spécialisation dont les limites apparaissent et traduisent une rupture évolutive rejoignant les prédictions et observations plus larges de sociologues, d'épistémologues et de philosophes (Morin et al 1993, Minc 1993, Serres 1991) sur l'évolution de la société.

Il nous est apparu logique, dans ce contexte de rupture et de crise complexe affectant l'élevage dans ses rapports aux filières et aux territoires, de nous référer à une représentation systémique et d'en adopter les postulats. Les réflexions engagées dans ce sens visent à stimuler des recherches respectant cette représentation, à partir du concept de système agraire et en y intégrant le contenu biologique et socioculturel des populations animales domestiquées.

Cette approche large de l'élevage et de la prise en compte de son support racial, sous une forme de rationalité procédurale, conduit à une révision de l'approche à fondement génétique et mécaniste qui prévaut dans la loi sur l'élevage. Elle place l'élevage au sein du développement rural et soulève de multiples interrogations auprès des parties prenantes. L'adaptation d'abord d'une gestion, à partir de «structures lourdes» (Gruson 1987), à des pôles sociaux dispersés dont les relations internes et pratiques sont mal connues : on sait que ce problème concerne bien d'autres structures du même type dans la Société industrielle.

Si l'intérêt de la race pour guider le développement des techniques dans une perspective spatiale et temporelle élargie apparaît de façon évidente, en dépit de toutes les difficultés, une interrogation demeure en l'absence de recherche plus précise. Malgré les travaux récents permettant de réduire l'intervalle de génération dans la mise en oeuvre de la sélection, les changements liés à cette dernière sont soumis à des délais et à une continuité qui garantit leur cumul entre générations successives. Or, l'incertitude et les changements accélérés d'orientation de l'élevage sont, à cet égard, inquiétants ; si les sélectionneurs avaient, dans le passé, des réflexes inhérents à leur fonction pour dépasser la conjoncture et anticiper les évolutions, l'introduction de ces facultés mentales dans les représentations des nouveaux acteurs sociaux, qui manifestent des attentes conjoncturelles fortes vis à vis de l'élevage, est pour le moins incertaine et sûrement redevable d'une longue pédagogie. La force des interrogations soulevées sur les structures lourdes de gestion de l'élevage doit être accompagnée, à cet égard, d'une grande prudence. En tout état de cause, ce texte ne visait pas à traiter de la sélection des races mais il ne pouvait en ignorer les fondements et les limites. Ce problème sera traité ultérieurement.

Nous avons fait état des critiques d'une démarche qui se voulait globale et systémique mais est restée appliquée au cas des populations bovines laitières. Les éleveurs de bovins sont aujourd'hui confrontés à des choix bien plus larges en ce qui concerne l'élevage de cette espèce et, plus largement, des ruminants. Malgré ces limitations nous avons simplement pris le parti de ce support pour étayer un mode de raisonnement.
Ce texte constitue une adaptation d'un article de l'auteur paru dans Nature, Sciences, Société, 1(4), 282297 , sous le titre "Société, race animale et territoire entre les théories et l'histoire : réflexion sur une crise». Il doit beaucoup à la rédaction et aux lecteurs de cette revue. Qu'ils soient remerciés pour leur aide. 


\section{Références bibliographiques}

Agabriel C. , Coulon J.B. , Marty G. , Bonaiti B. , Boniface P. , 1993. Effets respectifs de la génétique et du milieu sur la production et la composition du lait de vache. Etude en exploitations. INRA Prod. Anim., 6(3), 213-223.

Aglietta, 1981. Crises et transformations sociales. Problèmes économiques, 1723, 17-22.

Audiot A., Gibon A. ,Flamant J.C. , 1983. La conservation des races menacées : quels éleveurs? Ethnozootechnie, 33, 71-81.

Benoit M., 1993. Un indicateur des risques de pollution azotée nommé BASCULE (Balance azotée spatialisée des cultures de l'exploitation). Agriculture et qualité des eaux, 183-198. INRA Publications, Versailles.

Bertocchio F. , 1989. L'évaluation d'une race bovine à petit effectif : l'exemple de la race Béarnaise. Thèse de doctorat. Institut national polytechnique de Toulouse, $199 \mathrm{pp}+$ Annexes.

Boichard D., Bonaiti B. , Barbat A.,Briend M. 1992. Le modèle sous-jacent à l'évaluation des valeurs génétiques. INRA Prod. Anim. hors série «Eléments de génétique quantitative et application aux populations animales", 185-196.

Bonnemaire J. , Vissac B. , 1986. Races bovines et modèles de développement. In, Pour une agriculture diversifiée. M. Jollivet (éd.) L'Harmattan, coll. alternatives rurales, 252-267.

Brossier J., Vissac B., Le Moigne JL.(Ed), 1990. Modélisation systémique et système agraire. Décision et organisation. INRA Versailles, $365 \mathrm{pp}$

Cholley D. , 1946. Problèmes de structures agraires et d'économie rurale. Annales de géographie, 81-100.

Cristofini B. , Deffontaines J.P., Raichon C. , de Verneuil B., 1978. Pratiques d'élevage en Castagniccia. Exploration d'un milieu naturel et social en Corse. Etudes rurales, 71-72, 89-109.

Deffontaines J.P., 1972. Analyse régionale des systèmes de production agricole et "science du paysage». Position de recherche. Espace géographique $n^{\circ} 4$.

Deffontaines JP, Benoit M. , Brossier J., Chia E. , Gras F. , Roux M., 1993. Agriculture et qualité des eaux. Diagnostic et propositions pour un périmètre de protection. INRA Editions, Versailles.

Flamant J.C., Cabannes-Audiot A. , Vallerand F., 1989. Les populations humaines gestionnaires des populations animales. Colloque sur la gestion des ressources génétiques animales des espèces domestiques. Doc. INRA.

Geffroy B. , 1978. Industrialiser l'élevage bovin, cent ans d'utopie. Thèse de doctorat de 3ème cycle de sociologie rurale. Université de Paris X Nanterre.

Gilibert J. , 1991. Evolution des races, des modes de production et des types de fromages. Ethnozootechnie, 47, 83-98.

Gilibert J. ,Courbon D. , 1990. Développement régional : le niveau laiterie. Qualification des flux de lait. Symposium FEZ sur les systèmes d'élevage, Toulouse, $6 \mathrm{pp}$.
Gruson C., 1987. In : Vers une éthique politique Editions de la Maison des Sciences de l'Homme, Paris.

ITEB-INRA，1985，1986，1987，1988. Bilan d'indexation des reproducteurs bovins. Publication. ITEB-NIE.

Lauvergne J.J., 1979. Modèles de répartition des populations bovines domestiques animales après migration par vagues successives à partir d'un centre d’origine. Annales Génét. Sel. Anim. 11.

Legay J.M. , 1993. Commentaires. NSS 1(4), 298 299

Le Moigne JL., 1977-1984. La théorie du système général. Théorie de la Modélisation (2ème éd.), Presses Universitaires de France, Paris.

Madani T. , 1993. Complémentarité entre élevages et forêts dans l'Est algerien : fonctionnements et dynamiques des systèmes d'élevage dans le massif des Beni Salah. Thèse USTL Montpellier, 140pp + Ann.

Mazoyer M. , 1985. Rapport de synthèse au Comité Dynamique des Systèmes Agraires. Ronéoté, 16 pages.

Minc A., 1993. Le nouveau Moyen-Age. Ed. Gallimard, $249 \mathrm{pp}$.

Morin E., Kern B., 1993. Terre-Patrie. Editions du Seuil, Paris.

Perrot C. , 1991. Un système d'information construit à dire d'experts pour le conseil technico-économique aux éleveurs de bovins. Thèse de doctorat INAP-G, $215 \mathrm{pp}+$ Ann

Plet F. , 1991. Quotas et industrie laitière en France. In les exploitations agricoles et leur environnement, J. Brossier et E. Valceschini (eds). INRA Editions, 178-193.

Poutous M., Vissac B., 1962. Recherche théorique des conditions de rentabilité maximum de l'épreuve de descendance des taureaux d'insémination artificielle. Ann. Zootech., 11, 233-256.

Russel N. , 1986. Like engendring like : heredity and animal breeding in early modern England. Cambridge University Press, $271 \mathrm{p}$.

Serres M. 1991. La réception de Mr M. Serres à l'Académie Française. Le discours du récipiendaire, Le Monde, 3.4-2, pp 11-12.

Tirel J.C. , 1991. Agriculture : des voies nouvelles pour de nouveaux défis (ronéoté).

Vavilov N.J. , 1925. Studies on the original cultivated plants. Bulletin of applied botany and plant breeding, 16(2), pp 1-248.

Vissac B., 1968. Etude génétique de la race d'Aubrac, In : L'Aubrac : étude ethnologique, linguistique, agronomique et économique d'un établissement humain. I. CNRS, Paris, 27-101.

Vissac B. , 1978. L'animal domestique révélateur des relations entre la Société et son milieu. INRA, doc. département SAD. 
Vissac B. , 1983. Histoire agraire, mouvements et rythmes de l'élevage. In : De la touffe d'herbe au paysage. INRA publications, 313-336.

Vissac B. , 1989. Pratiques collectives et organisations de l'élevage. In : Les exploitations agricoles et leur environnement : essais sur l'espace technique et économique J. Brossier et E. Valceschini (eds) 83-100, INRA Editions, Paris.

Vissac B. , 1993. Société, race animale et territoire. Entre les théories et l'histoire : réflexions sur une crise. NSS 1(4), 282-297.
Vissac B. , Benoit M., 1993. Les formes d'agriculture au carrefour des filières et des territoires. Colloque Agricultures et Société, Association Descartes, pp 91-98.

Vissac B. , Bonnemaire J. , 1987. L'élevage dans le développement. In : Les agriculteurs et la politique depuis 1970. Colloque de la Société des Sciences politiques.

Vissac B. , Hengten A., 1979. Présentation du département de recherches sur les Systèmes Agraires et le Développement. INRA, doc département SAD.

\section{Summary}

Animal populations and agrarian systems. The case of dairy cattle in France.

This paper attempts to explore the crisis between society and territory as materialized in the area of livestock farming, and to propose new research paths. This crisis is expressed simultaneously by surplus production and depressed quality of output on one hand and by society's new perception of damage caused to the environment by livestock farming practices on the other hand. The case of dairy farming is exemplary for this purpose, since this industry has constantly been at the vanguard of modernity in livestock farming. The notion of agrarian system seemed to provide an appropriate means of approaching this complexity. That of animal population considered in its biological as well as cultural dimensions has been used as an indicator of the agrarian system.

The first part surveys the changes that have occurred in France over the last two centuries in dairy cattle populations and in the agrarian systems to which they were linked. Three entities are successively analysed and used to define the notion of animal population : local populations, breeds, specific lines derived from collective selection schemes.

The second part highlights the main features of these changes : extension of breed ranges and structuring of livestock organisations along with breed specialisation, regionally, supplanting of the older breeds by the more evolved breeds at a pace that depended on the evolution possibilities of agrarian systems, dissemination of new population types from focal points that developed on the margins of the range of older one's. However, simultaneously with this long term process, the older breeds were seen to resist locally. Interestingly, this resistence is being stimulated by the crisis.

To address this contradiction in the current evolution of dairy cattle farming, we need to broaden our reflection and include the concept of umetade velopment ${ }^{*}$ put forward by Morin. On the basis of a few examples, the bio-ecological, psychological and socio-cultural aspects of this evolution and their interactions are discussed.

We suggest that these aspects be integrated in an Action-Research procedure that would associate people involved in the production sectors with people charged with the management of rural territories in which animal populations could be the indicators of a local technical culture.

These new research perspectives should help refine the field of application of locally based multidisciplinary studies resting on the two postulates of comprehensiveness and complexity of livestock. dominated agrarian systems. They might contribute some elements for reconsidering the 1964 french livestock Act in which animal populations would retain a preeminent place.

VISSAC B., 1994. Populations animales et systèmes agraires. L'exemple des bovins laitiers. INRA Prod. Anim., 7 (2), 97-113. 